\title{
The Global Assessment of Nuclear Data, GANDR
}

\author{
D.W. Muir ${ }^{1}$, A. Trkov $^{2}$, I. Kodeli ${ }^{3}$, R. Capote ${ }^{4}$, and V. Zerkin ${ }^{4}$ \\ 1 International Atomic Energy Agency (Consultant), P.O. Box 452, Kittery, Maine, USA \\ 2 Jozef Stefan Institute, Ljubljana, Slovenia \\ 3 International Atomic Energy Agency representative at the OECD/NEA Data Bank, 92130 Issy-les-Moulineaux, France \\ 4 International Atomic Energy Agency, Nuclear Data Section, 1400 Vienna, Austria
}

\begin{abstract}
The GANDR project was initiated by the IAEA in 2001 with several ambitious goals, including the eventual provision of assistance to data experts in the planning of new measurements. We describe a hardware and software system developed in support of this project, now in operation in the IAEA Nuclear Data Section. A specific design goal of the system is to be able to track, in complete detail, the impact of new experiments on the uncertainties and correlations of 91000 independent parameters. This number is sufficient, for example, to describe the broad, energy-dependent features of most cross section types for 130 different target materials. Applications of GANDR to date have utilized experimental data from the EXFOR database. EXFOR includes only the diagonal elements of the data covariance matrix (variances), and often the supplied variances describe only the statistical part of the data uncertainty, so the GANDR system offer several options for modifying and supplementing the available experimental uncertainties.
\end{abstract}

\section{Introduction}

International organizations, including the International Atomic Energy Agency and the OECD Nuclear Energy Agency, have traditionally devoted considerable resources to supporting and coordinating nuclear data development work in their respective Member States. In the planning of data development activities, the most frequently asked question is "Are the existing data accurate enough?" If not, the next question usually is "What measurements should be performed to remedy the situation?"

In many important nuclear applications, neutron transport calculations must be performed in order to predict the values of practical quantities of interest, such as reactor control-rod worth, biological dose to accelerator operators, and activation of the components of a fusion reactor. In transport applications, questions like this are difficult to answer, because there is a very complex relationship between the accuracy of the basic input data and the accuracy of the output of the calculations.

Because of this complex relationship, research groups around the world have long recognized the potential contribution of sensitivity and uncertainty analysis, based on perturbation theory, to help address these questions, ref. [1]. However, past progress has been slowed by the need to track what has seemed to be an impossibly large amount of correlation information. With the remarkable developments that have taken place in computer capacities in recent years, this approach has become a much more interesting possibility.

In view of the need and these encouraging technological developments, in 2001 the IAEA initiated a project to develop a system based on sensitivity and uncertainty analysis for the Global Assessment of Nuclear Data Requirements (GANDR). In this paper, we present a report on the goals and status of the GANDR project, and we provide a technical description of a functioning hardware and software system, developed in support of the project, now in operation in the Nuclear Data Section.

Although the GANDR system is being developed with global data assessment in mind as the eventual application, the system has been shown to be adaptable to more specific near term needs as well. For example, the GANDR system was employed in recent evaluations of nuclear data and covariances of neutron-induced reactions up to $60 \mathrm{MeV}$ on thorium [2], protoactinium [3] and tungsten isotopes [4]. For these applications, the standard GANDR initialization of the data covariances with a non-informative prior was replaced with Monte Carlo calculated model covariances [5] reflecting nuclide-specific constraints imposed by nuclear physics.

In response to interest like this on the part of the data evaluation community, the full GANDR system (codes, associated data files, and complete documentation) has been made available on the IAEA Nuclear Data Centre web site. For details, see the Documentation section below.

\section{GANDR Project}

Reaching the future goal of providing data experts with guidance in experiment planning will require access to a complete and consistent evaluation of nuclear data covariances, based on the current state of data measurements. The formatted covariance data contained in the current generation of nuclear reaction data files do not provide a sufficiently uniform and consistent baseline for this purpose.

One problem is that the quality and completeness of the existing information is highly variable from material to material. A second major problem is that, by design, the covariances in the existing international data evaluations do not reflect the impact of available, high-quality integral data. The main reason for this is that the introduction of integral data would generate very strong cross-material correlations 
and would thereby greatly expand the volume of these widely disseminated data files.

Therefore, an important intermediate goal of the GANDR project is to perform a global evaluation of data covariances from first principles, within a unified evaluation framework. While consistency and wide coverage are both crucial, it is possible (and indeed necessary) to impose a certain level of coarseness on the actual evaluation process, as detailed below. Compromise is possible here because the goal is a practical tool for comparing the benefits of different experiments, not a complete characterization of all aspects of data uncertainty.

As planned, the global uncertainty evaluation will incorporate significant quantities of both high-quality differential data and clean integral data. By "clean", we mean data from simple-geometry integral experiments, preferably data that has been internationally recommended for the testing of differential data. Inclusion of integral data is important, because judgments concerning the need for new measurements should be based on all currently available experimental information, not just a portion of it.

An additional benefit of incorporating integral data into the GANDR evaluation process is that it reduces the need for exhaustive attention to all compiled differential data in this process. If accurate integral data are taken into account, older differential data with low accuracy will have no significant impact on the final data uncertainties and can safely be neglected.

\section{Scope of the evaluation}

In order to keep the evaluation task manageable, we make the simplifying assumption that the fine details of evaluated nuclear data, such as the shape of energy-dependent cross section in the neighbourhood of a single resonance, fine structure of neutron scattering angular distributions, and most emitted-particle angle-energy distributions, are sufficiently well constrained by nuclear physics that they can be treated as known quantities.

In contrast, nuclear physics provides relatively little guidance on the absolute magnitude of integrated neutron cross sections. The accuracy of such data rests ultimately on the absolute accuracy of experiments and, in many cases, on a relatively small number of measurements. Unfortunately, data measurements are susceptible to various types of systematic error, such as problems in the determination of the neutron flux, the sample size, the background or the detector efficiency.

In characterizing this uncertainty in integrated cross sections, we assume that the true value of each cross section of interest differs from the best existing evaluation by a smoothly varying multiplicative function $\mathrm{A}(\mathrm{E})$. These functions are defined on a fixed, 74-point energy grid that spans the range from $10^{-5} \mathrm{eV}$ to $150 \mathrm{MeV}$. The GANDR evaluation of data uncertainties is limited to determining the uncertainty of the nodal values of the functions $\mathrm{A}(\mathrm{E})$ as a function of material and reaction type.

For further economy, nuclear reactions are organized into 25 standard reaction types, some of which are groups of ENDF-format reaction types. For example, inelastic scattering to nuclear levels with excitation energies $1-6 \mathrm{MeV}$ is treated as a single reaction in the GANDR framework. Because of the existence of threshold reactions, we have found it sufficient to allocate 700 parameters to each target material.

Finally, "global" coverage is defined, for the present purposes, as including the data for 130 different target materials. We follow the lead of the large regional evaluations on the question of which materials to treat as elemental and which as isotopic. The total number of GANDR parameters is thus $700 \times 130$, or 91000 . For further details regarding the definition of the GANDR parameters, see tables 1-1 through 1-5 in Volume 1 of the GANDR report, ref. [6].

The covariance matrix describing the uncertainties of the parameters and their correlations thus contains $91000 \times$ $91001 / 2=4 \times 10^{9}$ unique numbers. Storage of a single copy of the complete GANDR covariance matrix requires, in double precision, a storage capacity of 33.1 gigabytes. In the current GANDR hardware environment, two separate copies of this large covariance matrix are maintained, on separate hard disk drives, one copy containing the current covariances and the other containing the covariances prior to the most recent update.

\section{ZOTTVL least squares solver}

Motivated by the considerations discussed above, an early focus of the GANDR project has been the development of a new generalized linear least squares solver. As a part of the GANDR project, the earlier IAEA evaluation program ZOTT99, ref. [7], has been modified extensively in order to treat problems having a much larger scope. The new, Very Large, version of ZOTT is ZOTTVL.

Now complete, ZOTTVL makes extensive use of datapaging techniques in order to handle the updating of the 91000 parameters, taking explicit account of the $4 \times 10^{9}$ covariances of these parameters. The data paging methodology relies heavily on partitioning the large matrices into smaller sub-matrices that reflect the inherent 700-parameter $\times 130$-material structure of the information. For additional details, see Volume 2 of the GANDR report, ref. [8].

Differential and integral data tend to group naturally into blocks of several hundred to several thousand in size, each block corresponding to one actual experimental setup, with strong data correlations within a block but with relatively weak correlations between blocks. Thus the array dimensioning in ZOTTVL accommodates blocks of generally-correlated experimental information that contain up to 3600 data points. The GANDR system can accommodate the input of an arbitrary number of such blocks of data.

Also as discussed in Volume 2, timing tests have been run with the new code. In these tests, a series of test problems were run in which every one of the GANDR parameters and covariances are updated, in response to a varying number of artificial integral data as input. It was found that, to a good approximation, the total running time on a Dell Precision 530 Workstation varies with the number of integral data $n b$ according to the following simple formula, holding for $n b$ up to about 1200: CPU time $=(n b+60)$ minutes. The constant term relates to the CPU time consumed in performing the 
significant task of transferring the 33 gigabytes of data from one disk drive into core and then back out to the other disk drive in each test problem. While these running times are certainly not trivial, these tests establish that it is practical to utilize generalized least squares to update this enormous data set on a modern personal computer.

The number of materials in the "GANDR Library" is an input parameter to ZOTTVL, so it is easy to switch from the full-library update mode, as we have just discussed, to a single-material mode simply by setting this input parameter equal to one. In the single-material mode, running times of ZOTTVL are not measured in hours but in minutes. All computer runs discussed in the remainder of this paper correspond to using ZOTTVL in the single-material mode.

\subsection{GANDR auxiliary programs}

In addition to ZOTTVL, we have written a pre-processor program GAPREP that reads GANDR relevant user input and prepares all of the input files required to update the parameters and covariances in response to a set of data measurements, using ZOTTVL as the least-squares solver.

Another important function of the GAPREP program is to assist the user in supplementing the uncertainty estimates from EXFOR (which contains only the diagonal of the data covariance matrix), with estimates of correlated uncertainty components, both of within an experiment and between experiments.

In addition, the program GAPREP includes features that support an iterative approach to solving moderately nonlinear least squares problems, as will be encountered later in the GANDR project. The method employed, Gauss-Newton iteration, takes into account the fact that sensitivities of integral data generally depend to some extent on the differential nuclear data being evaluated. This involves the management of a set of data files external to ZOTTVL, which is a purely linear solver.

In addition, a post-processor program GAPOST has been developed to display the results of each evaluation update, both as edited text listings and in publication-quality plots. See examples in figures 1 and 2 below. In order to enforce consistency between GAPREP and GAPOST, the codes share a library of low-level subroutines. These shared routines are stored on the GANDR master source file as "comdecks" that can be "called" by more that one program.

An additional small program GABROW browses the large GANDR binary data files and displays selected portions on a computer terminal. For more information on GAPREP, GAPOST and GABROW, see Volume 3, ref. [9].

The current GANDR system includes, in addition to the GANDR suite of computer programs, a Master EXFOR Library (MEL) of measured microscopic nuclear data. The MEL was created by extracting all cross section data in the international EXFOR database (as of May 2005) for the 130 GANDR materials. The MEL is stored in ASCII format for convenient access by the GANDR programs. See Volume 5 for details, ref. [10]. Also, a Master PENDF Library (MPL) has been created, using NJOY99.64 to process the ENDF/BVI.8 evaluations for the GANDR materials into resonance reconstructed, point-ENDF form.

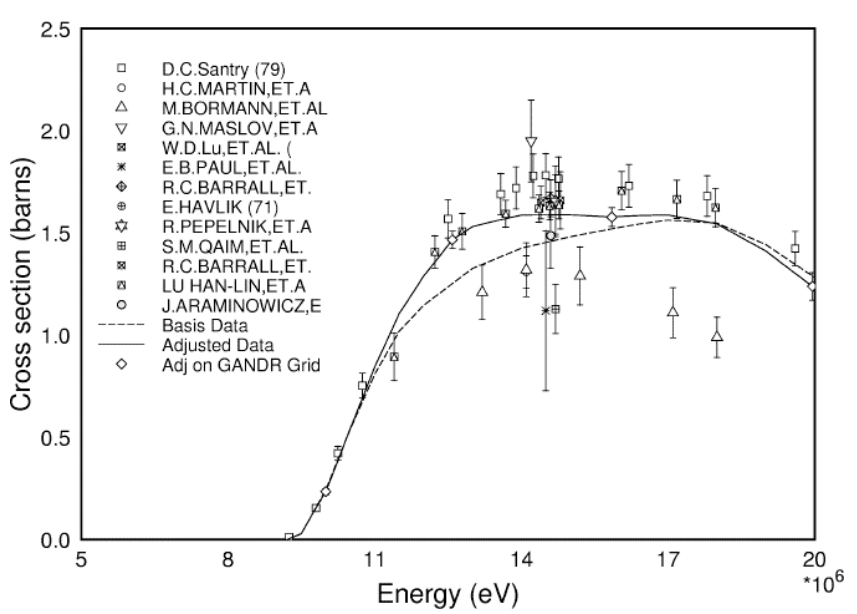

Fig. 1. ${ }^{127} \mathrm{I}(\mathrm{n}, 2 \mathrm{n})$ results with original uncertainties.

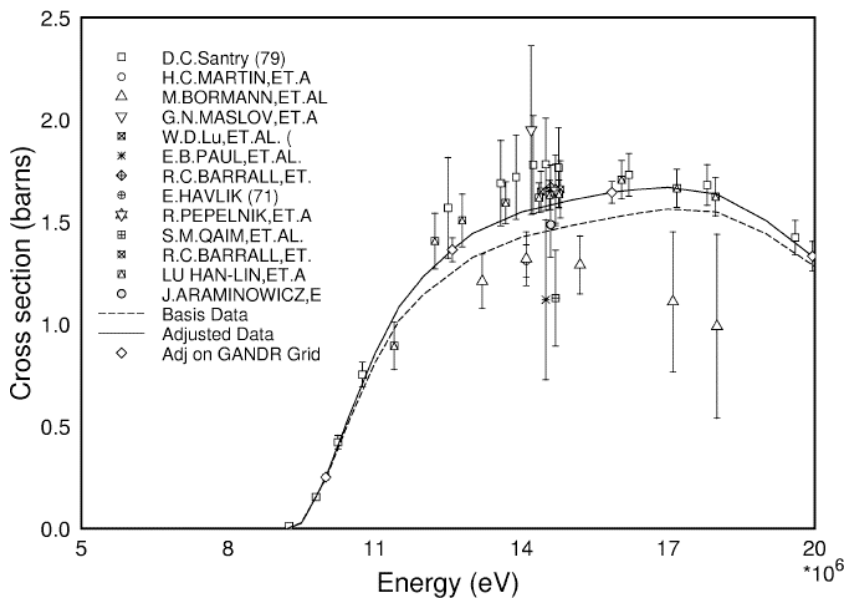

Fig. 2. ${ }^{127} I(n, 2 n)$ results with increased uncertainties for outliers.

With the GANDR system in its present form, the user can, relatively easily, select a material and reaction type and then combine data from ENDF and from EXFOR to obtain an estimate of the corresponding data covariances. Of course, the quality of this estimate will depend crucially on the quality of the EXFOR uncertainty values. To improve the quality may require a substantial effort by the user in reviewing and supplementing the EXFOR uncertainty information.

To illustrate a number of points made above, we show in figure 1 the result of applying the GANDR system to an evaluation of the cross section and uncertainties for the ${ }^{127} \mathrm{I}(\mathrm{n}, 2 \mathrm{n})$ reaction. The error bars shown in the graph, prepared by GAPOST, reflect the data uncertainties as they are given in the EXFOR library.

Two features are clear. First, the measurements can be divided into two groups, some higher than the ENDF evaluation (dashed line) and some lower. Second, the two groups differ from one another by more than the claimed uncertainties. The least squares solution (solid line) attempts to fit all of the data, primarily by making a fairly large increase in the cross section multiplier at $12.59 \mathrm{MeV}$.

In figure 2 we repeat the exercise, this time using the GANDR system default for treating measurement uncertainties. In this treatment, a "band of consistency" is considered to exist in the region defined by the reference evaluated curve, 


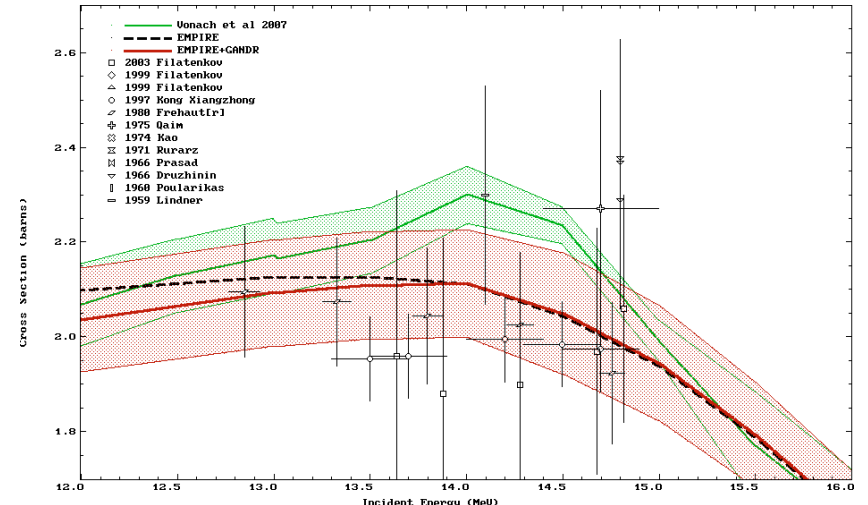

Fig. 3. Uncertainties in ${ }^{186} \mathrm{~W}(\mathrm{n}, 2 \mathrm{n})$.

plus or minus $7 \%$ of the reference. If the error bar of a given data point fails to overlap with this band of consistency, then the data point is considered an "outlier", and the size of the error bar is increased until it just touches the band boundary. In this example, nearly all of the data points in the EXFOR set qualify as outliers according to this test. Here, and in general, the effect of increasing the size of the error bars in this way is twofold. First, the final evaluated uncertainties are increased somewhat. See the points labelled "Adj. on GANDR Grid" (open diamond symbols). In addition, the evaluated data is much more faithful to the shape of the reference curve.

In figure 3 the experimental data and evaluated cross section curves for $(\mathrm{n}, 2 \mathrm{n})$ reaction on $\mathrm{W}-186$ nucleus are compared to the new evaluated curves before and after the adjustment with GANDR (labelled "EMPIRE" and "EMPIRE-GANDR", respectively).

The figure 3 shows that the model covariance prior is very effective in preserving a realistic shape of the obtained cross sections and that the generalised least squares method does not a priori lead to underestimation of uncertainties, provided that systematic uncertainties and correlations in the experimental data are correctly taken into account.

\section{Documentation}

To facilitate use of the GANDR system, we have produced a five-volume set of reports. These cover topics ranging from above mentioned theoretical and programming aspects to user input instructions and sample problems, ref. [11].

All of the above-mentioned information, including the reports, the Fortran programs, and the two Master Libraries, is available from the IAEA Nuclear Data Centre at http://wwwnds.iaea.org/gandr. The data libraries MEL and MPL are included on that site within the large data file gfiles.tar.gz (81 megabytes).

The authors wish to thank Mr. Liam Costello of the IAEA Nuclear Data Section for help and guidance in setting up the Linux environment and the web site for the GANDR project. Much of the work reported in this paper was performed under contractual agreements approved and supported by the IAEA Division of Physical and Chemical Applications.

\section{References}

1. L.N. Usachev, Yu.G. Bobkov, Perturbation Theory and Experiment Planning in Relation to Nuclear Data for Reactors, (in Russian) (Atomizdat, Moscow, 1980).

2. R. Capote et al., Evaluation of tungsten isotopes in the fast neutron range including cross-section covariance estimation (these proceedings).

3. A. Trkov, R. Capote, Cross-Section Covariance Data, Documentation of the Th-232 Evaluation for ENDF/B-VII.0 $(\mathrm{MAT}=9040 \mathrm{MF}=1 \mathrm{MT}=451)$, National Nuclear Data Center, Brookhaven National Laboratory (http://www.nndc.bnl.gov), 15 December 2006.

4. A. Trkov, R. Capote, Cross-Section Covariance Data, Documentation of the $\mathrm{Pa}-231$ and $\mathrm{Pa}-233$ Evaluations for ENDF/BVII.0 (MAT $=9133$ and $9137 \mathrm{MF}=1 \mathrm{MT}=451$ ), National Nuclear Data Center, Brookhaven National Laboratory (http://www.nndc.bnl.gov), 15 December 2006.

5. R. Capote et al., Extension of the Nuclear Reaction Model Code EMPIRE (these proceedings).

6. D.W. Muir, Global Assessment of Nuclear Data Requirements, Volume 1. Project Overview. Available online, see text.

7. D.W. Muir, ZOTT99, Data Evaluation using Partitioned Least Squares, Code package IAEA1371/01, NEA Computer Program Service, 1999.

8. D.W. Muir, Global Assessment of Nuclear Data Requirements, Volume 2. The ZOTTVL Program. Available online, see text.

9. D.W. Muir, Global Assessment of Nuclear Data Requirements, Volume 3. Auxiliary Programs GAPREP, GAPOST and GABROW. Available online, see text.

10. D.W. Muir, Global Assessment of Nuclear Data Requirements, Volume 5. Preparation of the EXFOR Master Library for the GANDR Project. Available online, see text.

11. D.W. Muir, Global Assessment of Nuclear Data Requirements, Volume 4. User Input Instructions and Five Sample Problems Based on EXFOR Data. Available online, see text. 